\title{
Atomic Scale Microstructure and Properties of Se-Deficient Two-Dimensional MoSe
}

\author{
Ossi Lehtinen, ${ }^{*}{ }^{\dagger}$ Hannu-Pekka Komsa, ${ }^{\ddagger}$ Artem Pulkin, ${ }^{\S}$ Michael Brian Whitwick, ${ }^{\perp}$ Ming-Wei Chen, ${ }^{\perp}$

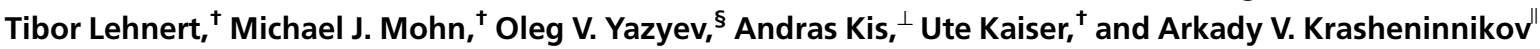 \\ ${ }^{\dagger}$ Group of Electron Microscopy of Materials Science, Central Facility for Electron Microscopy, University of Ulm, $89081 \mathrm{Ulm}$, Germany, ${ }^{\ddagger}$ COMP Centre of Excellence, \\ Department of Applied Physics, Aalto University, P.0. Box 11100, Fl-00076 Aalto, Finland, ${ }^{\S}$ Institute of Theoretical Physics, Ecole Polytechnique Fédérale de \\ Lausanne (EPFL), CH-1015 Lausanne, Switzerland, ${ }^{\perp}$ Electrical Engineering Institute, Ecole Polytechnique Fédérale de Lausanne (EPFL), CH-1015 Lausanne, \\ Switzerland, and "Department of Applied Physics, Aalto University, P.0. Box 11100, FI-00076 Aalto, Finland
}

\begin{abstract}
We study the atomic scale microstructure of nonstoichiometric two-dimensional (2D) transition metal dichalcogenide $\mathrm{MoSe}_{2-x}$ by employing aberration-corrected high-resolution transmission electron microscopy. We show that a Se-deficit in single layers of $\mathrm{MoSe}_{2}$ grown by molecular beam epitaxy gives rise to a dense network of mirror-twin-boundaries (MTBs) decorating the 2Dgrains. With the use of density functional theory calculations, we further demonstrate that MTBs are thermodynamically stable

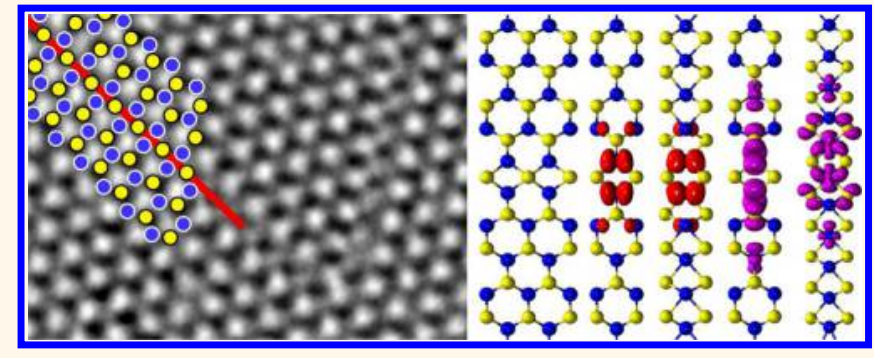
structures in Se-deficient sheets. These line defects host spatially localized states with energies close to the valence band minimum, thus giving rise to enhanced conductance along straight MTBs. However, electronic transport calculations show that the transmission of hole charge carriers across MTBs is strongly suppressed due to band bending effects. We further observe formation of MTBs during in situ removal of Se atoms by the electron beam of the microscope, thus confirming that MTBs appear due to Se-deficit, and not coalescence of individual grains during growth. At a very high local Se-deficit, the $2 \mathrm{D}$ sheet becomes unstable and transforms to a nanowire. Our results on Se-deficient $\mathrm{MoSe}_{2}$ suggest routes toward engineering the properties of 2D transition metal dichalcogenides by deviations from the stoichiometric composition.
\end{abstract}

KEYWORDS: 2D TMD · microstructure · stoichiometry $\cdot$ HRTEM $\cdot$ MBE $\cdot$ CVD $\cdot$ DFT $\cdot$ defects

A Itering the atomic composition of a material away from its perfect stoichiometric ratio is a standard approach for tailoring its properties. Perhaps the most famous example is the rich phase diagram of the $\mathrm{Fe}-\mathrm{Fe}_{3} \mathrm{C}$ system, where materials with diverse characteristics can be manufactured by varying the composition and the growth history. ${ }^{1}$ One can expect that the same approach should also work for two-dimensional (2D) systems. However, engineering of 2D materials by deviations from the 'perfect' stoichiometric balance between the primary components is a largely unexplored topic to this date.

This is particularly relevant to $2 \mathrm{D}$ transition metal dichalcogenides (TMDs). Recently, significant efforts have been directed toward producing 2D TMDs, due to their intriguing electronic properties, ${ }^{2}$ with variations of the chemical vapor deposition
(CVD) technique ${ }^{3-9}$ being the most promising in terms of large-scale production. The typical aim of such efforts has been the production of high-quality crystals at the perfect 1:2 stoichiometric composition, or 2D TMD random alloys ternary compounds ${ }^{10,11}$ with mixing either in the chalcogen ${ }^{12-14}$ or metal $^{15,16}$ sublattice. However, defects and dopant atoms can also be used to modify the properties of these materials. ${ }^{17-24} \mathrm{Re}$ cently, Kim et al. ${ }^{25}$ studied the influence of nonstoichiometry on the transport properties of CVD 2D MoS ${ }_{2-x}$ sheets and found increased mobilities in connection with a S-deficit, demonstrating the feasibility of engineering of 2D TMDs by moving away from the stoichiometric composition. Moreover, the thermoelectric properties, charge carrier concentrations ${ }^{26}$ and mass density ${ }^{27}$ of bulk $\mathrm{MoSe}_{2}$ are influenced by small deviations from the stoichiometric composition.
* Address correspondence to ossi.lehtinen@gmail.com.

Received for review January 19, 2015 and accepted March 6, 2015.

Published online 10.1021/acsnano.5b00410

(c) XXXX American Chemical Society 
Thus, as controlled composition engineering of the materials can take place only when the atomic scale morphology resulting from a given growth process can be predicted, microscopic information on the exact atomic structure of nonstoichiometric samples is essential for the optimization of growth process and complete understanding of the behavior of the new materials.

In terms of the phase diagram of the 2D TMDs, the reduced dimensionality imposes a strong limitation on the useful range of compositions; however only some distinct phases, such as the hexagonal $2 \mathrm{H}$, rhombohedral $3 \mathrm{R}$, and tetragonal $1 \mathrm{~T}$ structures of TMDs, are layered and thus can form stable/metastable 2D structures. ${ }^{28}$ Moving down in Se-content in the Mo-Se bulk system, for example, leads eventually to the crossing of the $\mathrm{MoSe}_{2}-\mathrm{Mo}_{3} \mathrm{Se}_{4}$ phase boundary and the crystal structure becoming three-dimensional.

Here, we explore the atomic scale microstructure of Se-deficient 2D MoSe $\mathrm{M}_{2-x}$ sheets, where the 2D topology is retained despite moving away from the stoichiometric composition. We investigate single layer 2D $\mathrm{MoSe}_{2-x}$ grown by molecular beam epitaxy (MBE) using aberration-corrected high-resolution transmission electron microscopy (AC-HRTEM) at $80 \mathrm{kV}$. The investigated material is found to be a nanocrystalline film with a $\mathrm{MoSe}_{1.983(1)}$ in-grain composition. The Mo-Se imbalance is manifested as mirror-twinboundaries (MTB) decorating individual grains, and the composition can be determined by measuring the areal density of the MTBs in AC-HRTEM images. Our calculations based on the density functional theory (DFT) indicate that such a configuration is energetically favorable in Se-deficient conditions. The calculated electronic band structure of the experimentally observed MTB presents evidence for localized states in a wide energy range in the semiconductor gap near the bottom of the conduction band. Our calculations of the ballistic electronic transport using the nonequilibrium Green's function approach across the MTB defect shows high transmission for the electron charge carriers, but low conductivity for the holes. We further demonstrate the formation of such boundaries in situ, when the electron beam of the TEM gradually removes Se atoms from the lattice and eventually the lattice spontaneously reorganizes locally into a mirror-domain. With further removal of Se, the 2D sheet is transformed into a nanowire, that is, a $2 \mathrm{D}$ to $1 \mathrm{D}$ transition is observed.

\section{RESULTS AND DISCUSSION}

Single layer 2D $\mathrm{MoSe}_{2}$ samples were grown in a customized MBE system, and the as-grown films were transferred onto TEM grids using poly(methyl methacrylate) (PMMA) as a temporary support, as described in more detail in the Materials and Methods section. The samples were then investigated in an aberration-corrected high-resolution transmission electron microscope (FEI Titan 80-300), operated at $80 \mathrm{kV}$.

At first impression, the sample was observed to consist of a free-standing membrane of single layer $\mathrm{MoSe}_{2}$ with a very fine grain-structure (only few small multilayer patches are present, as confirmed by extensive survey of the material using HRTEM, see Supporting Information Figure 4). A $130 \mathrm{~nm} \times 130 \mathrm{~nm}$ area is shown in Figure 1a with color coding applied to highlight grain distributions and orientations within the membrane (see Supporting Information Figure 1 and ref 29 for details on the generation of such an image). All the grains have the basal plane aligned with the membrane surface, but have seemingly random in-plane orientations, and the grains have diameters in the range of few tens of nanometers. The grains are stitched together by tilt-grain-boundaries consisting of rows of dislocations (Figure $1 \mathrm{~b}$ ), similar to what has been observed earlier for $\mathrm{MoSe}_{2},{ }^{9} \mathrm{MoS}_{2}{ }^{6,20}$ and graphene. $^{30-33}$ The small size of the grains can be tentatively explained by a high density of nucleation sites during growth of the membrane.

However, close examination of the images indicated that individual grains have additional structure. The grains consist of the regular trigonal $\mathrm{MoSe}_{2}$ lattice decorated by another type of very straight boundaries running through the grains, making sharp turns and occasionally forming closed triangular loops (these boundaries become also visible as a side effect of the orientation mapping, see Figure 1a). In Figure 1c, the positions of the Mo and the dual Se atoms are partly overlaid in the AC-HRTEM image, where the darkest spots correspond to the Se sites (i.e., dark atom contrast), the lighter spots to single Mo atoms, and the bright spots to the empty centers of the hexagons. On the basis of the identification of the atom positions (see also simulated image in Supporting Information Figure 5), the boundaries can be identified as mirrortwin-boundaries (MTBs), or, equivalently, as 60/180/ $300^{\circ}$ tilt grain boundaries, where the leading Se edges of two adjacent $\mathrm{MoSe}_{2}$ lattices meet (see Figure 1d for the structural model). With respect to edge type classification, we use the standard nomenclature. ${ }^{35}$ The Se- and Mo-edges are shown in Supporting Information Figure $4 \mathrm{a}$, and they are classified according to the atom types at the zigzag edge just after cutting the ideal lattice, independent of what kind of atoms are later on attached to the edge (e.g., the Mo edge may have zero, one or two Se atoms attached to the edge Mo atoms). The lattice on the opposite sides of these boundaries has seemingly the same orientation, but with an important distinction: the trigonal $\mathrm{MoSe}_{2}$ lattice is mirrored across the boundary, that is, the Mo and the dual Se sites are interchanged. While the Se-sublattice continues uninterrupted over the boundary, the Mo-sublattice has a discontinuity at the MTB resulting in Se-atoms at the boundary binding 


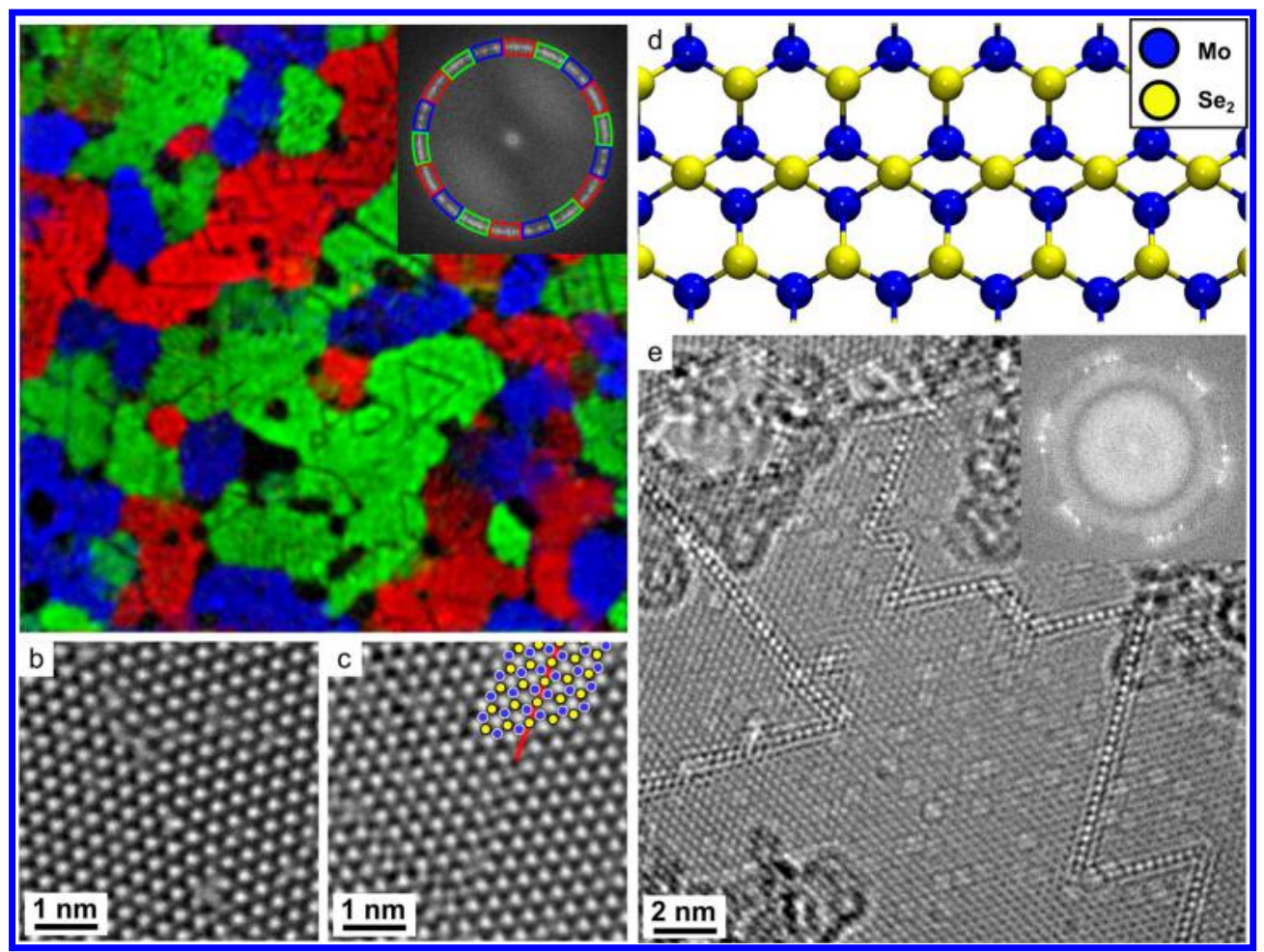

Figure 1. Atomic scale microstructure of a free-standing 2D Mo-Se layer imaged by AC-HRTEM. (a) The grain structure, with the color coding corresponding to different grain orientations. The map is generated in digital postprocessing, by selecting different orientations in the fast Fourier transform of the image and compositing the images (see Supporting Information Figure 1 for details). (b) A close-up view of a tilt grain boundary. The brighter lattice sites are Se vacancies and their concentration increases rapidly during imaging due to the electron beam. ${ }^{34}$ (c) A close-up view of a mirror-twin-boundary (MTB) embedded within a grain, with Mo and Se positions partly overlaid with circles. (d) A structural model of the MTB. (e) An image acquired at a special focus condition placing the $\mathrm{MoSe}_{2}$ lattice frequency at a minimum of the contrast transferfunction (see the inset FFT). At such imaging conditions, the MTBs become highly visible (the linear features), even when covered by contamination. The spots within the grains are Se vacancies.

to four Mo-atoms instead of three. ${ }^{9,36}$ No other types of MTBs were found in the sample. At various locations, a Se site appears lighter, which is due to either one or both of the Se atoms missing, and the number of such sites increases during imaging. These are Se vacancies produced by the electron beam of the microscope. ${ }^{34}$

MTBs have also been reported earlier in $\mathrm{MoSe}_{2}{ }^{9}$ and $\mathrm{MoS}_{2}{ }^{7,20}$ Recently, a very high density of MTBs was observed in a $\mathrm{MoSe}_{2}$ monolayer grown on top of graphene, ${ }^{37}$ and features in a $\mathrm{MoSe}_{2}$ thin film grown on top of a cleaved $\mathrm{MoS}_{2}$ surface ${ }^{38,39}$ can also be explained by MTBs. The structure of the MTBs is interpreted differently in ref 37 from what we observe here, but the uncorrected HRTEM images presented in that work do not allow unambiguous interpretation of the atomic structure, ${ }^{40}$ and in our opinion, it cannot be excluded that the structure of the boundaries is actually identical to what is observed here and in other reports on MTBs in $\mathrm{MoSe}_{2}{ }^{9}$

An important feature of an MTB, in the context of nonstoichiometry and atomic structure, is that the Modensity is higher at the boundary as compared to the perfect $\mathrm{MoSe}_{2}$ lattice, which has an interesting consequence: as the MTBs are observed in significant densities within the grains, a measurable deviation from the stoichiometric 1:2 ratio of Mo and Se is present, and the MTBs can be interpreted as an atomic scale feature resulting from the nonstoichiometry of the sample.

To quantify the density deviation, i.e., extract the exact composition of the grains, we acquired a large number of HRTEM images of the sample. The imaging was conducted in a nonstandard imaging mode (see Figure 1e). That is, when the objective lens is adjusted away from the 'optimal' Scherzer focus to a specific underfocus, where the spatial frequency of the $\mathrm{MoSe}_{2}$ lattice coincides with a zero-crossover in the contrasttransfer-function of the microscope, the image contribution of the continuous lattice is 'filtered' out and the discontinuities in the lattice become more visible, even when covered by contamination. Using such imaging conditions greatly improved the speed and reliability of the MTB mapping. It should be pointed out that a similar effect can be achieved through numerical filtering in the Fourier transform of the image in postprocessing.

A total area of $18700 \mathrm{~nm}^{2}$ was imaged and analyzed and within this area a total length of $1730 \mathrm{~nm}$ of MTBs was measured. The number of extra Mo atoms per unit length associated with a MTB is $1.00 \mathrm{Mo} / \mathrm{nm}$, and consequently, the Se to Mo ratio within the grains is determined to be 1:1.983(1) (assuming an uncertainty 
of $\pm 100 \mathrm{~nm}$ in the measured total MTB length). The mass density of the material was calculated to be $0.32(2) \%$ higher than perfect $\mathrm{MoSe}_{2}$, keeping in mind that the Mo density is increased at the MTBs. The tiltgrain-boundaries can be Mo- or Se-rich ${ }^{36}$ as well. However, our analysis is limited to the in-grain composition, excluding the edges of the grains, and thus the composition of the tilt-boundaries is not accounted for. As already mentioned, a high density of Se-vacancies is also visible in the HRTEM images. This can be attributed to generation of vacancies by the electron beam during imaging ${ }^{34}$ as discussed later, and is thus not accounted for in the composition determination of the pristine material.

Although observation of the atomic structure during growth is not possible, insights into the growth history and thus the origin of the MTBs can be gained by analyzing the observed structures. Two possible scenarios can be imagined for forming the MTBs during growth (postgrowth formation is discussed later). First, the observed type of MTBs can form when two adjacent grains merge. This requires (a) that the grains grow in the direction of the leading Se-edge, and (b) that the mistilt of the two merging grains is close to $180^{\circ}$ (due to the trigonal symmetry of the lattice, mistilts of $60^{\circ}$ and $300^{\circ}$ are equal to $180^{\circ}$ ). Second, the MTBs could form during the growth of individual grains, where, e.g., formation of MTBs would be initiated during the growth of the grain and the growth would continue with an MTB extending in the growth direction.

For assessing the validity of the first scenario, we can look at the distribution of the grain orientations, and the frequency at which MTBs are observed as compared to other tilt boundaries. No preferred orientation of individual grains is found (see Supporting Information Figure 2), which means we should have a uniform distribution of mistilt angles of the grain boundaries. Thus, the hyphothesis of MTB formation through merging of growing adjacent grains can be tested by looking at the mistilt angle distribution in the sample. According to earlier calculations, ${ }^{20}$ the grain boundary energy decreases within a $10^{\circ}$ deviation from the optimal $180^{\circ}$ orientation, and if we assume that grains meeting within this range reorient to optimize the energy and form MTBs, the expected fraction of MTBs among all grain boundaries is one in six. However, a density of $0.097(6) \mathrm{nm} / \mathrm{nm}^{2}$ of tilt-grain boundaries is observed in the sample, which is almost identical to the density of MTBs $\left(0.093(5) \mathrm{nm} / \mathrm{nm}^{2}\right)$, meaning that the MTBs are overrepresented. For a one-to-one ratio between other tilt-boundaries and MTBs, formation of MTBs should take place at up to $\pm 30^{\circ}$ deviation from the ideal $180^{\circ}$ tilt, and even if few degree mistilts over the MTBs are occasionally seen in the sample (see for example the lower left MTB in Figure 1e), high mistilts over MTBs of up to $30^{\circ}$ are never observed. On the basis of this, formation of the MTBs simply due to merging of misoriented neighboring grains can be ruled out.
As formation due to merging of grains does not seem to be a plausible explanation for the appearance of the MTBs, we need to look into the second scenario: Formation of MTBs during the growth of individual grains. Here, formation of MTBs would be initiated during the growth of the grain and the growth would continue with an MTB extending in the growth direction. As shown below by our DFT calculations, moving to Mo-rich growth conditions significantly lowers the formation energy of MTBs, giving rise to an increased density during growth. Also, Se has a low sticking coefficient, ${ }^{41,42}$ which can result in a local Se-deficit even at excess deposition rates. Locations in the sample with multiple MTBs radially emanating from the same point in a star like pattern are observed (see Supporting Information Figure 3a), which can be interpreted as nucleation sites of the grains, supporting the hyphothesis of formation of MTBs during grain growth in the growth direction. Currently, precise control of this process is not possible, however, and further work is necessary to enable controlled growth of MTBs embedded in larger crystallites.

An additional insight into the growth history can be gained from the boundary statistics: as MTBs where the leading Mo-edges of the grains meet (see the structures $4-6$ in Figure 2a) are never observed in the sample, the grain growth can be deduced to have taken place in the leading Se-edge direction. As discussed in more detail in the Supporting Information, our DFT calculations show that, indeed, the environment influences the energetics of different edge terminations, with the leading Se-edge being the lowest energy configuration in a Mo-rich environment, while the leading Mo-edge is preferred at Se-rich conditions (see Supporting Information for details).

Until now, we have observed a specific kind of atomic scale microstructure associated with a deviation from the perfect 1:2 Mo-Se ratio in the sample, namely MTBs embedded within the grains. To learn whether this is actually the preferred microstructure within the 2D Mo-Se phase diagram when moving down in Se content from the stoichiometric 1:2 ratio, we conducted DFT calculations, determining the energetics of a number of defect structures with a Se-deficit.

We start by considering the relative energies of various types of MTBs to find out whether the MTBs observed in the experiments correspond to those having the lowest energy, or if formation is determined by other factors, i.e., kinetics of the growth process. The energies and geometries of the candidate structures are shown in Figure 2a,b and classified as forming either from two Mo- or Se-edges facing each other (see further discussion in Supporting Information). Structure (1) corresponds to the one observed in our samples, (2) was observed $\mathrm{in}^{43}$ in $\mathrm{MoS}_{2}$, (3) and (6) were suggested in ref $44,(4)$ was proposed in ref 37 and is analogous to (1) with $\mathrm{Mo} / \mathrm{Se}_{2}$ sites swapped, and a structure similar to (5) 


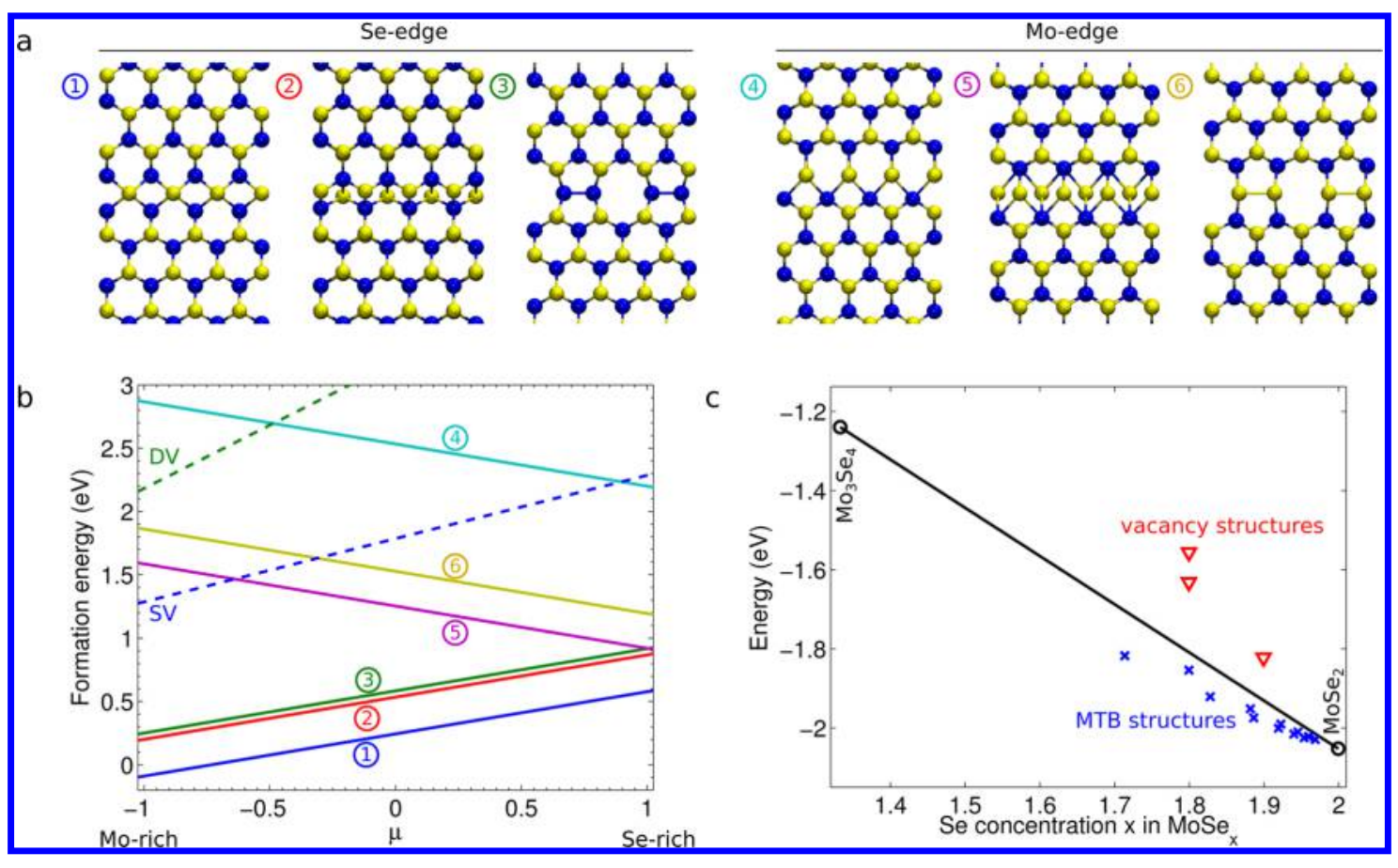

Figure 2. Calculated energetics of twin boundary structures (a) Atomic structures of the MTB candidates considered. The structures are grouped depending on whether they are constructed from Mo- or Se-edges. (b) The calculated formation energies of all MTBs as well as single (SV) and double vacancy (DV) lines as a function of the Se chemical potential. (c) Heats of formation of defective $\mathrm{MoSe}_{2}$ systems compared to the energies of the bulk $\mathrm{MoSe}_{2}$ and $\mathrm{Mo}_{3} \mathrm{Se}_{4}$ phases. The chemical potentials for $\mathrm{Mo}$ and Se are taken from the reference phases. The calculated energies are scaled to the number of Mo atoms in the system and plotted as a function of their Se/Mo fraction.

was suggested in ref 36 (although we found that our structure with the two domains shifted by half a lattice constant along the boundary is lower in energy by $0.6 \mathrm{eV})$. The calculations are carried out in a ribbon geometry. The formation energy is defined as

$$
E^{f}=E(\mathrm{MTB})-\left(n_{\mathrm{Mo}} \mu_{\mathrm{Mo}}+n_{\mathrm{Se}} \mu_{\mathrm{Se}}+2 \gamma\right)
$$

where $E(\mathrm{MTB})$ is the total energy from the supercell calculation and $\gamma$ is the edge energy (see Supporting Information). $n_{\mathrm{Se}}$ and $n_{\mathrm{Mo}}$ are the numbers of Se and Mo atoms in the supercell. Chemical potentials $\mu_{\mathrm{Mo}}$ and $\mu_{\mathrm{Se}}$ are determined through the condition of the equilibrium with $\mathrm{MoSe}_{2}: \mu_{\mathrm{Mo}}+2 \mu_{\mathrm{Se}}=E\left(\mathrm{MoSe}_{2}\right)$ (where $E\left(\mathrm{MoSe}_{2}\right)$ is the energy from a primitive cell of $\left.\mathrm{MoSe}_{2}\right)$, and limited by the Mo bulk phase in the Mo-rich end and by the $\mathrm{Se}_{8}$ molecule on the Se-rich end. Among the candidates, structure (1) has the lowest energy by a fairly large margin of $0.3 \mathrm{eV}$, but all MTB structures constructed from Se-edges seem to be favored over those with Mo-edges. We also estimated the MTB formation energy by constructing supercells with triangular mirror domains (similar to that shown in Figure 4). The formation energy for structure (1) at the Mo-rich limit is $0.12 \mathrm{eV}$, about $0.2 \mathrm{eV}$ higher than that from the ribbon calculation, which can be assigned to differences in relaxation and on the position of the Fermi-level in the system.

We also considered vacancy line structures, similar to what we have previously observed in $\operatorname{MoS}_{2}{ }^{19,45}$ where the lines were found to be energetically favorable, as compared to isolated vacancies. Their formation energies are also shown in Figure $2 \mathrm{~b}$ but are found to be much higher than those for the MTBs for the whole range of chemical potentials.

Reports in literature concerning the phase diagram of $\mathrm{Mo} / \mathrm{Se}$ systems are scarce. $\mathrm{MoSe}_{2}$ and $\mathrm{Mo}_{3} \mathrm{Se}_{4}$ are known to be stable phases, but the behavior at the intermediate stoichiometries appears to be unknown. ${ }^{46}$ Thus, the question, whether the MTB-rich structures are lower in energy than a system with coexisting $\mathrm{MoSe}_{2} / \mathrm{Mo}_{3} \mathrm{Se}_{4}$ phases, needs to be answered. In Figure 2c, we plot heat of formation $H$ for all these systems as a function of their Se/Mo content. As usual, it is defined as $H=E-n_{\mathrm{Mo}} \mu_{\mathrm{Mo}}-n_{\mathrm{Se}_{\mathrm{e}}} \mu_{\mathrm{Se}}$, where $E$ is the total energy of the system composed from $n_{\text {Mo }}$ molybdenum and $n_{\mathrm{Se}}$ selenium atoms, and $\mu_{\mathrm{Mo}}$ and $\mu_{\mathrm{Se}}$ are the chemical potentials of Mo and Se atoms in the reference phases. Clearly, the structures where Se-deficiency is due to a large concentration of MTBs are favored over systems consisting of a combination of $\mathrm{MoSe}_{2}$ and $\mathrm{Mo}_{3} \mathrm{Se}_{4}$ phases. For the case of $\mathrm{MoSe}_{2}$ structures with large concentration of vacancies, the situation is different-they are even higher in energies than the mixed phases. Thus, we propose that Se-deficiency in $\mathrm{MoSe}_{2}$ under thermal equilibrium manifests itself in the formation of MTBs and consequently in a large density of inversion domains.

We also analyzed the electronic structure of MTBs, Figure 3. Two bands predominantly localized at the 


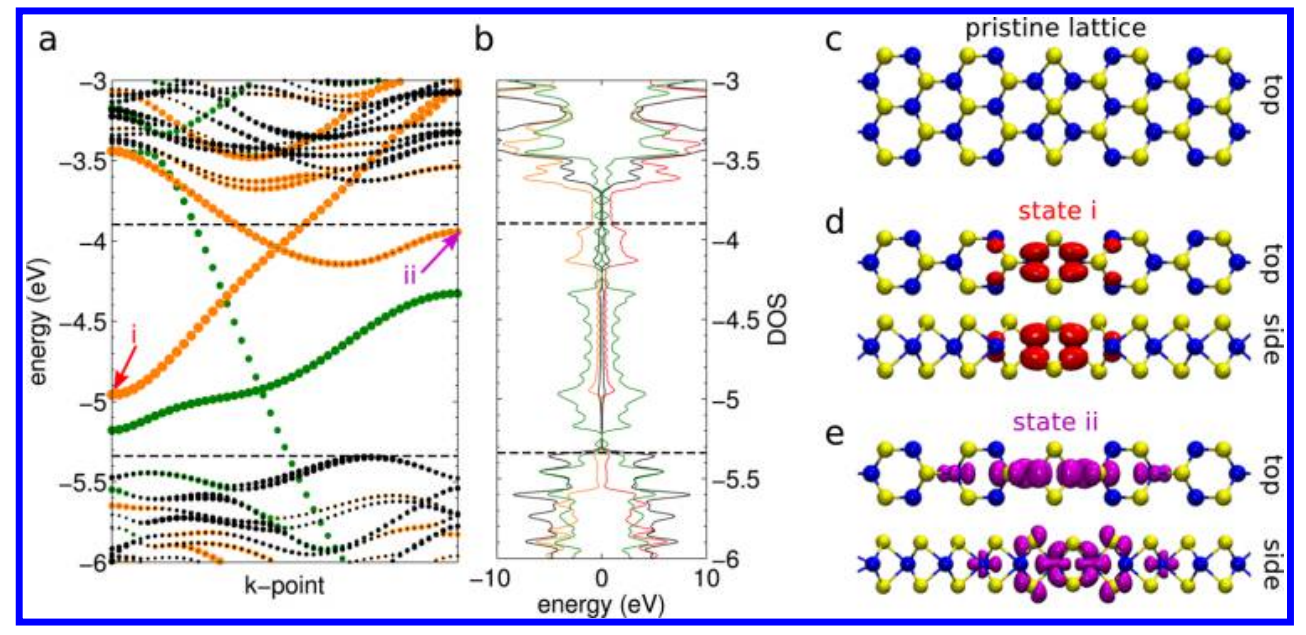

Figure 3. Atomic and electronic structures and density of states of the MTB. (a) The band structure and (b) corresponding density-of-states from a ribbon calculation with one MTB. The states are colored by the projection to different regions of the system (orange, near MTB region; green, edge region; black, bulk region). (c-e) The atomic structure of the MTB system together with the charge density isosurface visualization of wave functions of the two MTB-localized states (i) and (ii) within the gap.

MTB can be distinguished. The squared absolute value of the wave functions indicate that both are derived from the surrounding Mo atoms. The additional density of states brought in from these bands covers a wide energy range within the gap that could be suggestive of higher conductivity along the MTBs, at least for straight periodic boundaries. In a more realistic picture, one has to include also backscattering of 1D conductance channels on the turns and junctions of domain boundaries. There is a chance that such scattering effects would considerably reduce the conductance due to the extra channels. Suppression of localized electronic states at the turns of periodic grain boundaries has recently been observed in epitaxial polycrystalline graphene grown on the SiC substrate. ${ }^{47}$ Indeed, our electrical measurements (to be published in another article) performed on the samples showed no improvement, either due to this effect or to the high density of tilt-grain-boundaries in the sample, which may dominate the transport characteristics of the sample due to increased scattering. ${ }^{7}$

To gain further insight into the transport properties, we performed nonequilibrium Green's function calculations of the transmission of ballistic charge carriers across the MTBs. Figure 4a shows the momentum- and energy-resolved transmission probabilities. For the electron charge carriers ( $E>1.45 \mathrm{eV}$ ), we find only moderately reduced transmission which is likely to be governed by the resonant scattering involving the localized states discussed above. For the holes $(E<$ $0 \mathrm{eV}$ ), however, we observe much stronger suppression of the transmission. As one can see from the right panel in Figure 4a, the momentum-averaged transmission $\langle T(E)\rangle$ is exponentially decreasing with decreasing the energy of the hole charge carriers. This behavior can be ascribed to the potential bending at the MTB defect, which is clearly exposed in Figure 4b. The nonstoichoimetric composition of the defect results in downward

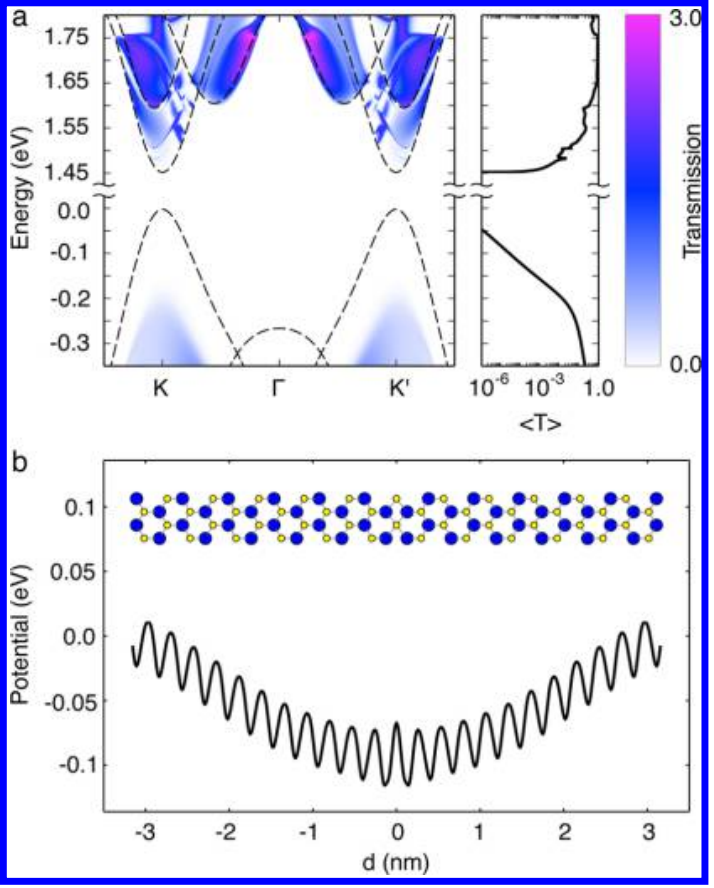

Figure 4. Electronic transport across the MTB defect. (a) Momentum and energy-resolved ballistic charge-carrier transmission probabilities across the MTB. The contours of electronic bands of pristine monolayer $\mathrm{MoSe}_{2}$ are shown as dashed lines with the energy of the valence band maximum set as reference. Right panel presents the momentum averaged transmission $\langle T\rangle$ on the logarithmic scale. (b) The calculated self-consistent Hartree potential along the transport direction and averaged over the planes perpendicular it. Corresponding structure of an MTB defect is presented on the same scale.

bending of electrostatic potential by $c a .0 .1 \mathrm{eV}$ in a relatively wide region around the defect. Such potential bending acts as an effective barrier for the lowenergy hole charge carriers, while its effect on the electron charge carriers is much less pronounced.

In addition to imaging MTBs in the as-grown samples, formation of mirror-domains was observed also in situ 


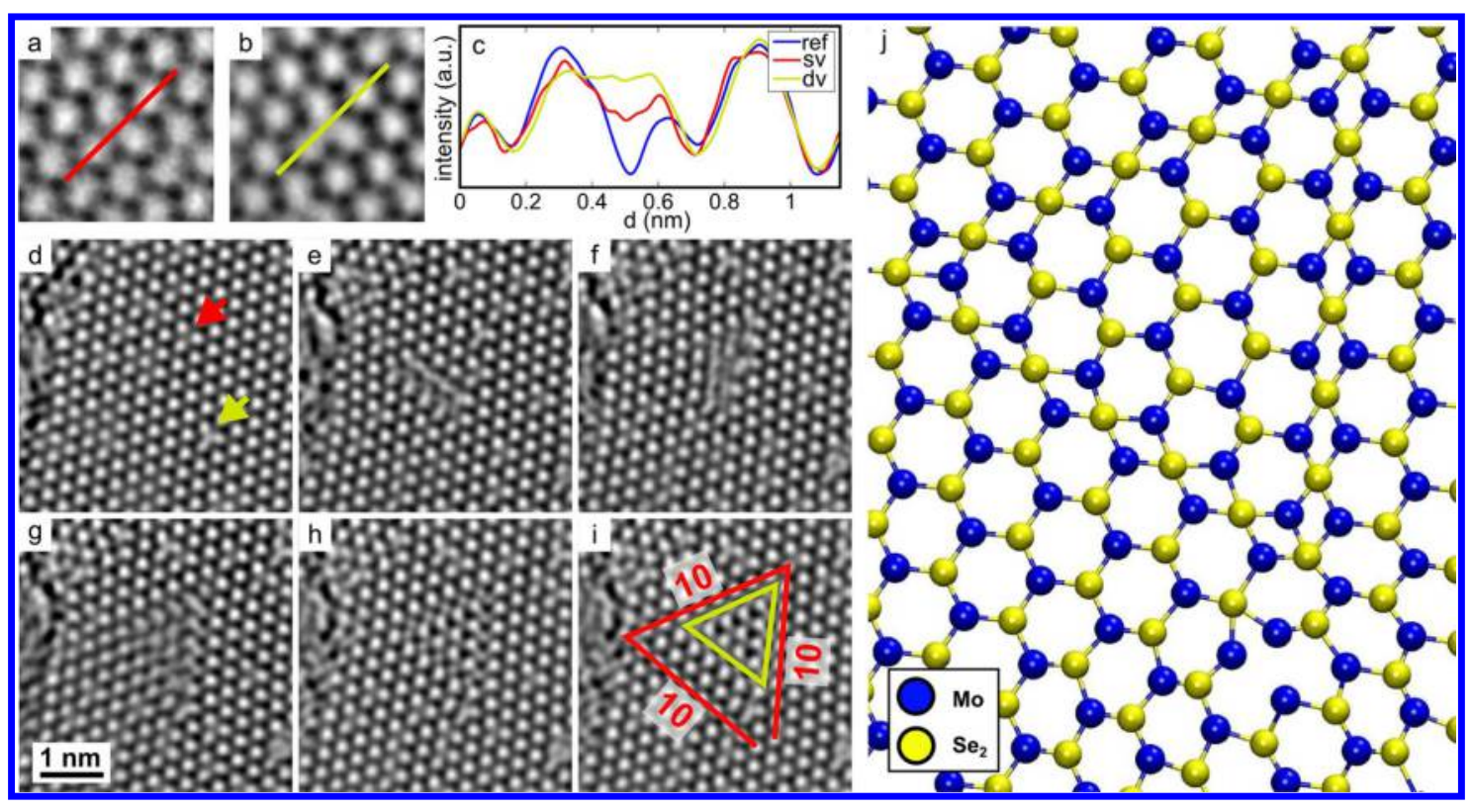

Figure 5. Evolution of 2D MoSe $e_{2}$ during $80 \mathrm{kV}$ electron irradiation. (a and b) A single and double Se vacancy induced by the electron beam. (c) Line profiles measured across the single (red line) and double (yellow line) vacancies, with a line profile over pristine $\mathrm{MoSe}_{2}$ lattice as a reference (blue line). (d) An area in $\mathrm{MoSe}_{2}$, where a number of isolated vacancies are present. (e) Part of the vacancies have aggregated into a linear structure. (f) The vacancy line has reoriented itself. (g) A frame, during which many atoms within a triangular area are in motion, rendering the exact structure indiscernible. (h) A mirror-twin-domain has appeared, accompanied by an edge dislocation. (i) Frame $h$, with the yellow lines overlaid denoting the mirror-twinboundary, and red lines with the length of 10 unit cells, indicating the presence of a dislocation. (j) The atomic structure of the newly formed mirror-twin-domain.

under the beam due to production of Se vacancies under the $80 \mathrm{keV}$ irradiation (see Figure 5, panels a and b, for examples of single and double Se vacancies, respectively, with corresponding line-profiles in panel c, and Supporting Information Figure 5 for simulated images). Due to the relatively high mass of Se, the removal of the Se atoms cannot be explained by direct knock-on damage, ${ }^{19,34}$ and should be attributed to inelastic processes such as ionization/radiolysis ${ }^{48,49}$ or beammediated chemical etching. ${ }^{50}$ By measuring the rate at which vacancies are formed, and taking the electron dose rate into account, a vacancy production cross section of 3.9(4) barn was determined, which is comparable to the value of 4.5(4) obtained earlier for freestanding $\mathrm{MoS}_{2}{ }^{48}$ at $80 \mathrm{keV}$. The exact experimental conditions, such as beam intensity, quality of the vacuum and sample preparation may influence the damage rates, however, as even the absence of any electron beam damage at $80 \mathrm{keV}$ in $\mathrm{MoS}_{2}$ has been reported. ${ }^{51}$

Evolution of a selected area in a $\mathrm{MoSe}_{2}$ is presented in Figure $5 d-i$. In frame $d$, numerous isolated vacancies produced under the electron beam are already visible. During continuous exposure, the number of vacancies increases and the vacancies migrate to form a linear vacancy aggregate (frame e). Subsequently, the vacancy aggregate changes orientation in frame $f$. In frame $g$, a reorganization of the lattice inside a triangular area is underway, resulting in a locally blurred image, which makes the atomic structure indiscernible. In frame $h$, the crystal has settled into a new stable configuration, i.e., a small mirror-twin-domain and a neighboring edge dislocation (the dislocation is visible already in frame g), while the number of Se-vacancies within the area has decreased. In panel (i) the new mirror-domain and a path of $3 \times 10$ lattice steps around the dislocation are overlaid on the micrograph of panel $h$, verifying the presence of the dislocation. An atomic model of the interpreted final structure is shown in panel (j). This sequence lends further support to the hypothesis that a MTB is the energetically preferred structure in the presence of a Se-deficit.

Supporting Information Movie 1 shows the evolution of another sample area over a $1 \mathrm{~min}$ time interval at a dose rate of $3.7 \times 10^{6} \mathrm{e} /\left(\mathrm{nm}^{2} \mathrm{~s}\right)$. An increasing number of vacancies are generated which migrate under the beam to first form linear vacancy aggregates, and later multiple small mirror domains. Also growth of the domains and migration of the MTBs are observed during the exposure. The large mirror domain at the lower left corner is present from the beginning of the imaging, and is assumed to have formed during the growth of the material. The slightly different appearance of the lattice at the opposite sides of the boundary can be attributed to residual $A_{2}$ astigmatism. ${ }^{40}$

Formation of an edge dislocation is a prerequisite for the formation of a mirror-twin-domain inside a preexisting $\mathrm{MoSe}_{2}$ crystal when the formation path is via removal of Se atoms (as opposed to formation during the growth of the crystal). When the mirror domain is formed, the Se-vacancies are eliminated, and surplus 
Mo is introduced at the MTBs. The extra atoms are attained by 'pulling' the neighboring crystal lattice toward the new mirror domain, which leads to the formation of the dislocation. In the presented case, a hole in the $\mathrm{MoSe}_{2}$ crystal close to the newly created dislocation allowed the dislocation to form without the formation of a complementary oppositely oriented dislocation, which would be required in the general case of a continuous crystal. Importantly, closed MTB loops without an associated dislocation are observed in the as-grown samples (see Supporting Information Figure $3 b$ ), which precludes formation of such domains in the pristine sample from Se vacancies after growth.

Many of the MTBs do not form closed loops, however, and thus, this kind of analysis is not possible in all the cases. Still, appearance of the MTBs observed already at the beginning of the imaging due to electron beam removal of Se does not seem likely. First, the mirror boundaries are in place already when imaging at low magnifications, and consequently, low dose-rates/Se vacancy concentrations. Second, the mirror domains and MTBs formed under the electron beam have much smaller dimensions and are typically connected to other extensive disorder in the lattice as compared to the relatively long and regular MTBs observed from the beginning, suggesting a different origin. Supporting Information Movie 1 shows the generally disordered state of the lattice at the time when the new mirror domains start appearing due to the electron beam.

Another comprehensive reorganization of the crystal structure takes place when moving further down in Se content during prolonged irradiation. A 2D to 1D transition takes place when neighboring growing holes form constrictions in the suspended layer and eventually the material self-organizes into nanowires (see Figure $6 a, b)$, which has been observed also earlier in $\mathrm{MoSe}_{2}{ }^{34}$ and $\mathrm{MoS}_{2}{ }^{52}$ The structural formula of the wires is MoSe, as confirmed by DFT calculations (the relaxed structural model is shown in Figure $6 c, d$ ) and HRTEM images simulations (Figure 6e, as simulated by the QSTEM software package ${ }^{53}$ ). In the context of the $2 \mathrm{D} \mathrm{MoSe}$ phase diagram, this event can be viewed as crossing the 2D-1D phase boundary on the Se-poor side.

\section{CONCLUSIONS}

In conclusion, using aberration-corrected highresolution transmission electron microscopy, and atomistic calculations, we have investigated the morphology of a Se-deficient 2D MoSe ${ }_{2-x}$ grown by molecular beam epitaxy, at the atomic scale. The Se-Mo

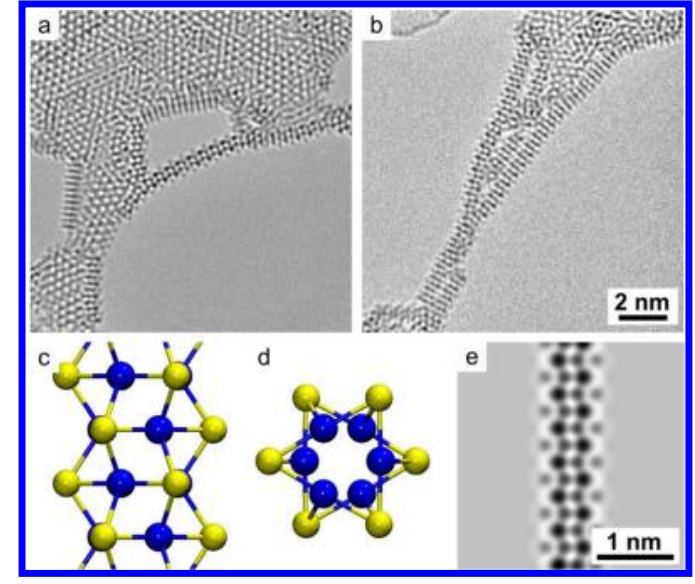

Figure 6. 2D to 1D transition resulting from prolonged electron irradiation. ( $a$ and $b$ ) AC-HRTEM images of MoSe nanowires suspended over holes in the $2 \mathrm{D} \mathrm{MoSe} \mathrm{M}_{2}$ layer. (c and d) Side and end projection of a DFT relaxed structural model of the nanowire. (e) Simulated HRTEM image of the nanowire.

imbalance was observed to be manifested as linear mirror-twin-boundaries decorating the individual grains in the film. The exact composition of the grains was measured to be $\mathrm{MoSe}_{1.983(1)}$ and the total density of the material was determined to be $0.032(2) \%$ higher than the density of perfect $\mathrm{MoSe}_{2}$. On the basis of density functional theory calculations, the observed structures were concluded to be thermodynamically stable in the case of a Se-deficit. Wide midgap states were predicted to be associated with the mirror-twinboundaries. The ballistic transmission of charge carriers across the MTB defects is only moderately affected in the case of electrons, but strongly suppressed for the holes as a result of potential bending effect. In situ formation of mirror-domains during gradual removal of Se atoms by the electron beam was also observed, which supports the prediction that the mirror-twinboundaries are the preferred structure in the case of a Se-deficit. Finally, a 2D to 1D transformation was found in the case of a high Se-deficit, corresponding to the crossing of the boundary of the 2D phase.

Our results show how the atomic scale microstructure of the binary 2D TMDs can be manipulated by the means of varying the exact composition. However, further work is required to attain control over the precise growth conditions to be able to produce the materials at exact predetermined compositions and crystal domain sizes. Drawing a parallel to the long tradition of materials science of bulk solids, this would open up vast possibilities for engineering the properties of this new class of 2D systems.

\section{MATERIALS AND METHODS}

Single layer $\mathrm{MoSe}_{2}$ was grown by a customized MBE system from Omicron Co. Our substrate was $270 \mathrm{~nm}$ silicon dioxide $\left(\mathrm{SiO}_{2}\right)$ on $525 \mu \mathrm{m}$ n-type $\mathrm{Si}$ chips $(12 \mathrm{~mm} \times 12 \mathrm{~mm})$. The substrates were outgassed at $300{ }^{\circ} \mathrm{C}$ in the preparation chamber and were transferred to the growth chamber. The growth was carried out at $450{ }^{\circ} \mathrm{C}$. A Kundsen cell and an electron beam source were used for Selenium (Se) and Molybdenum (Mo) evaporation, respectively. The flux was calibrated and measured by the quartz crystal microbalance 
with a Mo/Se flux ratio 1:30. Reflection high-energy electron diffraction (RHEED) was used to monitor in situ the formation of the crystalline structure during growth. Continuous liquid nitrogen was flowing during the ramping temperature and growth phase, with a base pressure of growth chamber at $\sim 10^{-10} \mathrm{mB}$.

To prepare samples for TEM characterization, the as-grown films were first coated with $\sim 500 \mathrm{~nm}$ PMMA, and were cured at $180^{\circ} \mathrm{C}$ for $5 \mathrm{~min}$. PMMA-embedded films were then lifted off in $30 \% \mathrm{KOH}_{(a q)}$ at $60^{\circ} \mathrm{C}$. The samples were then transferred into a deionized water (DI) bath, and TEM grids were put underneath in order to well-attach the floating films. The grids with the attached films atop were then baked at $60{ }^{\circ} \mathrm{C}$ to gently vaporize water and also to relieve wrinkles. After completely drying out, samples were dipped into acetone to remove PMMA residues and were rinsed by $\mathrm{DI}$ afterward.

AC-HRTEM characterization was conducted using an FEI Titan 80-300 microscope equipped with an image side hexapole spherical aberration corrector. The microscope was operated at $80 \mathrm{kV}$, and the extraction voltage of the field emission gun was lowered to $2 \mathrm{kV}$ in order to reduce the energy spread of the beam. The spherical aberration coefficient was corrected to $<20 \mu \mathrm{m}$, and the imaging was done at negative defocus, resulting in atoms appearing as dark spots.

Density-functional theory calculations were carried out in the plane-wave basis and within the projector-augmented wave (PAW) description of the core regions, as implemented in the VASP code. ${ }^{54,55}$ We adopt the exchange-correlation functional proposed by Perdew, Burke, and Ernzerhof (PBE). ${ }^{56}$ A 500 eV cutoff and $12 \mathrm{k}$-points along the periodic dimension(s) yield converged total energies. Our calculations accounted for the spin polarization while the spin-orbit interaction was neglected. The ballistic charge-carrier transmission was calculated using the nonequilibrium Green's function technique within the DFT framework as implemented in OpenMX code. ${ }^{5,58}$ The Hamiltonian was expressed in the localized pseudoatomic basis set (Mo:s2p2d2f1, Se:s2p1d1f1). The length of rectangular scattering region containing the relaxed MTB defect was set to $6.5 \mathrm{~nm}$ along the transport direction.

Conflict of Interest: The authors declare no competing financial interest.

Acknowledgment. O.L, T.L, M.J.M., and U.K. acknowledge financial support by the DFG (German Research Foundation) and the Ministry of Science, Research and the Arts (MWK) of Baden-Württemberg in the frame of the SALVE (Sub-Angstrom Low-Voltage Electron Microscopy) project and the DFG SPP 1459 "Graphene" project. O.L. acknowledges financial support by the Finnish Cultural Foundation. The work was carried out with partial financial support from the Academy of Finland through Project No. 263416. H.-P.K. further thanks the Academy of Finland for the support through its Centres of Excellence Programme (2012-2017) under Project No. 251748. We also thank CSC-IT Center for Science Ltd. and Aalto Science-IT project for generous grants of computer time. A.P. and O.V.Y. acknowledge support by the Swiss NSF (Grant No. PPOOP2_133552) and ERC project "TopoMat" (Grant No. 306504). Electronic transport calculations have been performed at the Swiss National Supercomputing Centre (CSCS) under project s515. M.B.W, M.-W.C and A.K. acknowledge support by the ERC grant "Flatronics" (Grant No. 240076).

Supporting Information Available: Description of the grain mapping procedure based on HRTEM images, and analysis of grain orientations. HRTEM images of a MTB nucleation site and a closed MTB loop. HRTEM images of multilayer patches. HRTEM image simulations of MTBs and Se vacancies. Details of the computation of the energetics of flake edges and MTBs. Comparison of formation energies of defect structures at different chemical potentials. Formation energies for various vacancy and vacancy aggregate structures. A video showing the evolution of a sample area during electron beam exposure. This material is available free of charge via the Internet at http://pubs.acs.org.

\section{REFERENCES AND NOTES}

1. Callister, W. D.; Rethwisch, D. G. Materials Science and Engineering: An Introduction; John Wiley \& Sons, Inc., New York, 2009.

2. Jariwala, D.; Sangwan, V. K.; Lauhon, L. J.; Marks, T. J.; Hersam, M. C. Emerging Device Applications for Semiconducting Two-Dimensional Transition Metal Dichalcogenides. ACS Nano 2014, 8, 1102-1120.

3. Kim, K. K.; Hsu, A.; Jia, X.; Kim, S. M.; Shi, Y.; Hofmann, M.; Nezich, D.; Rodriguez-Nieva, J. F.; Dresselhaus, M.; Palacios, T.; et al. Synthesis of Monolayer Hexagonal Boron Nitride on Cu Foil Using Chemical Vapor Deposition. Nano Lett. 2012, 12, 161-166.

4. Zhan, Y.; Liu, Z.; Najmaei, S.; Ajayan, P. M.; Lou, J. Large-Area Vapor-Phase Growth and Characterization of $\mathrm{MoS}_{2}$ Atomic Layers on $\mathrm{a} \mathrm{SiO}_{2}$ Substrate. Small 2012, 8, 966-971.

5. Shi, Y.; Zhou, W.; Lu, A.-Y.; Fang, W.; Lee, Y.-H.; Hsu, A. L.; Kim, S. M.; Kim, K. K.; Yang, H. Y.; Li, L.-J. Van der Waals Epitaxy of $\mathrm{MoS}_{2}$ Layers Using Graphene As Growth Templates. Nano Lett. 2012, 12, 2784-2791.

6. Najmaei, S.; Liu, Z.; Zhou, W.; Zou, X.; Shi, G.; Lei, S.; Yakobson, B. I.; Idrobo, J.-C.; Ajayan, P. M.; Lou, J. Vapour Phase Growth and Grain Boundary Structure of Molybdenum Disulphide Atomic Layers. Nat. Mater. 2013, 12, 754-759.

7. van der Zande, A. M.; Huang, P. Y.; Chenet, D. A.; Berkelbach, T. C.; You, Y.; Lee, G.-H.; Heinz, T. F.; Reichman, D. R.; Muller, D. A.; Hone, J. C. Grains and Grain Boundaries in Highly Crystalline Monolayer Molybdenum Disulphide. Nat. Mater. 2013, 12, 554-561.

8. Wang, X.; Gong, Y.; Shi, G.; Chow, W. L.; Keyshar, K.; Ye, G.; Vajtai, R.; Lou, J.; Liu, Z.; Ringe, E. Chemical Vapor Deposition Growth of Crystalline Monolayer MoSe 2 . ACS Nano 2014, 8, 5125-5131.

9. Lu, X.; Utama, M. I. B.; Lin, J.; Gong, X.; Zhang, J.; Zhao, Y.; Pantelides, S. T.; Wang, J.; Dong, Z.; Liu, Z. Large-Area Synthesis of Monolayer and Few-Layer $\mathrm{MoSe}_{2}$ Films on $\mathrm{SiO}_{2}$ Substrates. Nano Lett. 2014, 14, 2419-2425.

10. Komsa, H.-P.; Krasheninnikov, A. V. Two-Dimensional Transition Metal Dichalcogenide Alloys: Stability and Electronic Properties. J. Phys. Chem. Lett. 2012, 3, 3652-3656.

11. Kutana, A.; Penev, E. S.; Yakobson, B. I. Engineering Electronic Properties of Layered Transition-Metal Dichalcogenide Compounds Through Alloying. Nanoscale 2014, 6, 5820-5825.

12. Mann, J.; Ma, Q.; Odenthal, P. M.; Isarraraz, M.; Le, D.; Preciado, E.; Barroso, D.; Yamaguchi, K.; von Son Palacio, G.; Nguyen, A. 2-Dimensional Transition Metal Dichalcogenides with Tunable Direct Band Gaps: $\operatorname{MoS}_{2(1-x)} \mathrm{Se}_{2 \mathrm{x}}$ Monolayers. Adv. Mater. 2014, 26, 1399-1404.

13. Li, H.; Duan, X.; Wu, X.; Zhuang, X.; Zhou, H.; Zhang, Q.; Zhu, X.; Hu, W.; Ren, P.; Guo, P. Growth of Alloy $\mathrm{MoS}_{2 x} \mathrm{Se}_{2(1-X)}$ Nanosheets with Fully Tunable Chemical Compositions and Optical Properties. J. Am. Chem. Soc. 2014, 136, 37563759.

14. Gong, Y.; Liu, Z.; Lupini, A. R.; Shi, G.; Lin, J.; Najmaei, S.; Lin, Z.; Elías, A. L.; Berkdemir, A.; You, G. Band Gap Engineering and Layer-By-Layer Mapping of Selenium-Doped Molybdenum Disulfide. Nano Lett. 2014, 14, 442-449.

15. Chen, Y.; Xi, J.; Dumcenco, D. O.; Liu, Z.; Suenaga, K.; Wang, D.; Shuai, Z.; Huang, Y.-S.; Xie, L. Tunable Band Gap Photoluminescence from Atomically Thin TransitionMetal Dichalcogenide Alloys. ACS Nano 2013, 7, 4610-4616.

16. Su, S.-H.; Hsu, Y.-T.; Chang, Y.-H.; Chiu, M.-H.; Hsu, C.-L.; Hsu, W.-T.; Chang, W.-H.; He, J.-H.; Li, L.-J. Band Gap-Tunable Molybdenum Sulfide Selenide Monolayer Alloy. Small 2014, 10, 2589-2594

17. Radisavljevic, B.; Radenovic, A.; B, J.; G, V.; K, A. Single-Layer $\mathrm{MoS}_{2}$ Transistors. Nat. Nanotechnol. 2011, 6, 147-150.

18. Eda, G.; Yamaguchi, H.; Voiry, D.; Fujita, T.; Chen, M.; Chhowalla, M. Photoluminescence from Chemically Exfoliated $\mathrm{MoS}_{2}$. Nano Lett. 2011, 11, 5111-5116.

19. Komsa, H.-P.; Kotakoski, J.; Kurasch, S.; Lehtinen, O.; Kaiser, U.; Krasheninnikov, A. V. Two-Dimensional Transition Metal Dichalcogenides Under Electron Irradiation: Defect Production and Doping. Phys. Rev. Lett. 2012, 109, 035503. 
20. Zhou, W.; Zou, X.; Najmaei, S.; Liu, Z.; Shi, Y.; Kong, J.; Lou, J.; Ajayan, P. M.; Yakobson, B. I.; Idrobo, J.-C. Intrinsic Structural Defects in Monolayer Molybdenum Disulfide. Nano Lett. 2013, 13, 2615-2622.

21. Cheng, Y. C.; Zhu, Z. Y.; Mi, W. B.; Guo, Z. B.; Schwingenschlögl, U. Prediction of Two-Dimensional Diluted Magnetic Semiconductors: Doped Monolayer $\mathrm{MoS}_{2}$ Systems. Phys. Rev. B 2013, 87, 100401.

22. Ly, T. H.; Chiu, M.-h.; Li, M.-y.; Zhao, J.; Perello, D. J.; Cichocka, M. O.; Oh, H. M.; Chae, S. H.; Jeong, H. Y.; Yao, F. Observing Grain Boundaries in CVD-Grown Monolayer Transition Metal Dichalcogenides. ACS Nano 2014, 8, 11401-11408.

23. Yazyev, O. V.; Chen, Y. P. Polycrystalline Graphene and Other Two-Dimensional Materials. Nat. Nanotechnol. 2014, 9, 755-767.

24. McDonnell, S.; Addou, R.; Buie, C.; Wallace, R. M.; Hinkle, C. L. Defect-Dominated Doping and Contact Resistance in $\mathrm{MoS}_{2}$. ACS Nano 2014, 8, 2880-2888.

25. Kim, I. S.; Sangwan, V. K.; Jariwala, D.; Wood, J. D.; Park, S.; Chen, K.-S.; Shi, F.; Ruiz-Zepeda, F.; Ponce, A.; Jose-Yacaman, $M$. Influence of Stoichiometry on the Optical and Electrical Properties of Chemical Vapor Deposition Derived $\mathrm{MoS}_{2}$. ACS Nano 2014, 8, 10551-10558.

26. Conan, A.; Goureaux, G.; Zoaeter, M. Transport Properties of $\mathrm{MoTe}_{2-X}$ and $\mathrm{MoSe}_{2-X}$ Compounds between 130 and $300^{\circ}$ K. J. Phys. Chem. Solids. 1975, 36, 315-320.

27. Spiesser, M.; Marchal, C.; Rouxel, J. Sur Des Phases Molybdene-Selenium Non Stoechiometriques. Compt. Rend. Acad. Sci. 1968, 266, 1583-1586.

28. Wang, Q. H.; Kalantar-Zadeh, K.; Kis, A.; Coleman, J. N.; Strano, M. S. Electronics and Optoelectronics of TwoDimensional Transition Metal Dichalcogenides. Nat. Nanotechnol. 2012, 7, 699-712.

29. Mohn, M.; Lehtinen, O. A Grain Mapping Plugin for the ImageJ Software. https:/github.com/mmohn/OrientationMapping/, 2015. Online accessed Feb 25, 2015.

30. Grantab, R.; Shenoy, V. B.; Ruoff, R. S. Anomalous Strength Characteristics of Tilt Grain Boundaries in Graphene. Science 2010, 330, 946-948.

31. Yazyev, O. V.; Louie, S. G. Topological Defects in Graphene: Dislocations and Grain Boundaries. Phys. Rev. B 2010, 81, 195420.

32. Huang, P. Y.; Ruiz-Vargas, C. S.; van der Zande, A. M.; Whitney, W. S.; Levendorf, M. P.; Kevek, J. W.; Garg, S.; Alden, J. S.; Hustedt, C. J.; Zhu, Y. Grains and Grain Boundaries in Single-Layer Graphene Atomic Patchwork Quilts. Nature 2011, 469, 389-392.

33. Kurasch, S.; Kotakoski, J.; Lehtinen, O.; Skakalova, V.; Smet, J.; Krill, C. E.; Krasheninnikov, A. V.; Kaiser, U. Atom-By-Atom Observation of Grain Boundary Migration in Graphene. Nano Lett. 2012, 12, 3168-3173.

34. Lin, J.; Cretu, O.; Zhou, W.; Suenaga, K.; Prasai, D.; Bolotin, K. I.; Cuong, N. T.; Otani, M.; Okada, S.; Lupini, A. R.; Idrobo, J.-C.; et al. Flexible Metallic Nanowires with SelfAdaptive Contacts to Semiconducting Transition-Metal Dichalcogenide Monolayers. Nat. Nanotechnol. 2014, 9, 436-442.

35. Bollinger, M. V.; Jacobsen, K. W.; Norskov, J. K. Atomic and Electronic Structure of $\mathrm{MoS}_{2}$ Nanoparticles. Phys. Rev. B 2003, 67, 085410.

36. Zou, X.; Liu, Y.; Yakobson, B. I. Predicting Dislocations and Grain Boundaries in Two-Dimensional Metal-Disulfides from the First Principles. Nano Lett. 2013, 13, 253-258.

37. Liu, H.; Jiao, L.; Yang, F.; Cai, Y.; Wu, X.; Ho, W.; Gao, C.; Jia, J.; Wang, N.; Fan, H. Dense Network of One-Dimensional Midgap Metallic Modes in Monolayer $\mathrm{MoSe}_{2}$ and Their Spatial Undulations. Phys. Rev. Lett. 2014, 113, 066105.

38. Mori, T.; Abe, H.; Saiki, K.; Koma, A. Characterization of Epitaxial Films of Layered Materials Using Moire Images of Scanning Tunneling Microscope. Jpn. J. Appl. Phys. 1993, 32, 2945.

39. Murata, H.; Koma, A. Modulated STM Images of Ultrathin $\mathrm{MoSe}_{2}$ Films Grown on $\mathrm{MoS}_{2}(0001)$ Studied by STM/STS. Phys. Rev. B 1999, 59, 10327-10334.
40. Lehtinen, O.; Geiger, D.; Lee, Z.; Whitwick, M. B.; Chen, M.-W.; Kis, A.; Kaiser, U. Numerical Correction of Anti-Symmetric Aberrations in Single HRTEM Images of Weakly Scattering 2D-Objects. Ultramicroscopy 2014, 10.1016/j.ultramic.2014.09.010.

41. Tribollet, B.; Rayane, D.; Benamar, A.; Melinon, P. Study of Selenium Clusters. Z. Phys. D: At., Mol. Clusters 1992, 24, 87-93.

42. Hanna, G.; Mattheis, J.; Laptev, V.; Yamamoto, Y.; Rau, U.; Schock, H. Influence of the Selenium Flux on the Growth of $\mathrm{Cu}(\mathrm{In}, \mathrm{Ga}) \mathrm{Se}_{2}$ Thin Films. Thin Solid Films 2003, 431432, 31-36.

43. Zhou, W.; Zou, X.; Najmaei, S.; Liu, Z.; Shi, Y.; Kong, J.; Lou, J.; Ajayan, P. M.; Yakobson, B. I.; Idrobo, J.-C. Intrinsic Structural Defects in Monolayer Molybdenum Disulfide. Nano Lett. 2013, 13, 2615-2622.

44. Enyashin, A. N.; Bar-Sadan, M.; Houben, L.; Seifert, G. Line Defects in Molybdenum Disulfide Layers. J. Phys. Chem. C 2013, 117, 10842-10848.

45. Komsa, H.-P.; Kurasch, S.; Lehtinen, O.; Kaiser, U.; Krasheninnikov, A. V. From Point to Extended Defects in Two-Dimensional $\mathrm{MoS}_{2}$ : Evolution of Atomic Structure Under Electron Irradiation. Phys. Rev. B 2013, 88, 035301.

46. Bergmann, H.; Czeska, B.; Haas, I.; Wandner, K.-H. In Gmelin Handbook of Inorganic and Organometallic Chemistry; Katscher, H., Mohsin, B., Eds.; Springer-Verlag: Berlin and Heidelberg $\mathrm{GmbH}$; 1995; Supplement volume B, 8 .

47. Tison, Y.; Lagoute, J.; Repain, V.; Chacon, C.; Girard, Y.; Joucken, F.; Sporken, R.; Gargiulo, F.; Yazyev, O. V.; Rousset, S. Grain Boundaries in Graphene on SiC(0001) Substrate. Nano Lett. 2014, 14, 6382-6386. , PMID: 25330353

48. Algara-Siller, G.; Kurasch, S.; Sedighi, M.; Lehtinen, O.; Kaiser, U. The Pristine Atomic Structure of $\mathrm{MoS}_{2}$ Monolayer Protected from Electron Radiation Damage by Graphene. Appl. Phys. Lett. 2013, 103, 203107.

49. Zan, R.; Ramasse, Q. M.; Jalil, R.; Georgiou, T.; Bangert, U.; Novoselov, K. S. Control of Radiation Damage in $\mathrm{MoS}_{2}$ by Graphene Encapsulation. ACS Nano 2013, 7, 10167-10174.

50. Meyer, J. C.; Eder, F.; Kurasch, S.; Skakalova, V.; Kotakoski, J.; Park, H.; Roth, S.; Chuvilin, A.; Eyhusen, S.; Benner, G. An Accurate Measurement of Electron Beam Induced Displacement Cross Sections for Single-Layer Graphene. Phys. Rev. Lett. 2012, 108, 196102.

51. Garcia, A.; Raya, A. M.; Mariscal, M. M.; Esparza, R.; Herrera, M.; Molina, S. I.; Scavello, G.; Galindo, P. L.; Jose-Yacaman, M.; Ponce, A. Analysis of Electron Beam Damage of Exfoliated $\mathrm{MoS}_{2}$ Sheets and Quantitative HAADF-STEM Imaging. Ultramicroscopy 2014, 146, 33-38.

52. Liu, X.; Xu, T.; Wu, X.; Zhang, Z.; Yu, J.; Qiu, H.; Hong, J.-H.; Jin, C.-H.; Li, J.-X.; Wang, X.-R. Topdown Fabrication of SubNanometre Semiconducting Nanoribbons Derived from Molybdenum Disulfide Sheets. Nat. Commun. 2013, 4, 1776.

53. Koch, C. Determination of Core Structure Periodicity and Point Defect Density Along Dislocations, Ph. D. Thesis, Arizona State University,2002.

54. Kresse, G.; Furthmüller, J. Efficient Iterative Schemes for $a b$ Initio Total-Energy Calculations Using a Plane-Wave Basis Set. Phys. Rev. B 1996, 54, 11169-11186.

55. Kresse, G.; Joubert, D. From Ultrasoft Pseudopotentials to the Projector Augmented-Wave Method. Phys. Rev. B 1999, 59, 1758-1775.

56. Perdew, J. P.; Burke, K.; Ernzerhof, M. Generalized Gradient Approximation Made Simple. Phys. Rev. Lett. 1996, 77, 3865.

57. Ozaki, T. Variationally Optimized Atomic Orbitals for LargeScale Electronic Structures. Phys. Rev. B 2003, 67, 155108.

58. Ozaki, T.; Kino, H. Numerical Atomic Basis Orbitals from $\mathrm{H}$ to Kr. Phys. Rev. B 2004, 69, 195113. 